\title{
An experimental online dust measurement system for environmental control and emissions monitoring
}

\author{
M. Menacer ${ }^{1} \&$ M. Al-Mohammadi ${ }^{2}$ \\ ${ }^{I}$ Department of Computer Science, University of Taibah, \\ Al Madinah, KSA \\ ${ }^{2}$ Department of Mechanical Engineering, University of Nottingham, UK
}

\begin{abstract}
Particle pollution is the term for a mixture of solid particles and liquid droplets suspended in the ambient air. Research studies have associated exposure to elevated levels of these particles in the air with damaging health effects. Now, there is an increased regulatory pressure to demonstrate continuous compliance with microscopic particulate mass emission limits. This paper describes the various settings of an experimental project aimed at developing the principals of a non-intrusive device, using the Particle Image Velocimetry (PIV) laser technique, for online measurement of dust particulates. This study will allow the assessment and analysis of small particulates suspended in a slow moving flow medium (the simulation is performed in water), and to study the various aspects affecting particle size measurements.

Keywords: environmental control, laser application, PIV technique, particle measurement, emissions monitoring.
\end{abstract}

\section{Introduction}

Particle pollution (also called Particulate Matter (PM)) contains a mixture of microscopic solids particles or liquid droplets. It includes "inhalable coarse particles," with diameters ranging from 2.5 micrometers $(\mu \mathrm{m})$ to $20 \mu \mathrm{m}$ and "fine particles" with diameters equal to or smaller than $2.5 \mu \mathrm{m}$. The size of particles is directly linked to their potential for causing health problems. Small particles less than $20 \mu \mathrm{m}$ in diameter pose the greatest problems, because they can get deep 
into your lungs, and some may even get into your bloodstream, and can cause serious health problems [1].

Now, there are an increased regulatory pressure to demonstrate continuous compliance with microscopic particulate mass emission limits (Pollution), in coal power stations, chemical plants, Airplanes oil discharge (Kiyoto Treaty). Thus, the great desire to monitor online dust particulate size distribution. However, due to the complex nature of these measurements, currently, there are intrusive devices that measure a limited size range of dust particulates. Most of these devices require manual calibration that is necessary for accurate readings.

This experimental project aims at setting up the principals of a non-intrusive device, using the Particle Image Velocimetry (PIV) laser technique, for online environmental control and emissions monitoring that continuously measures dust particulate. PIV technique has been employed to aid the visualisation processes and provides analysis of qualitative and quantitative information about the spatial structure of fluid flows [2]. This technique has good accuracy, resolution and reliability for the instantaneous qualitative and quantitative measurements of a whole flow field. The principle of the PIV method is to determine the flow velocity from the motion of tracer particles (solid emissions in this study). A plane sheet of a laser light illuminates the flow area of interest and the light scattered from the tracer particles is recorded by means of a high resolution CCD camera. The velocity and particulate information is then extracted from images exposed to single, double or multiple laser pulses [3].

This paper describes the various settings put in place and the various data collected for analysis in order to achieve this goal. The proper equipments have been chosen to enable the assessment and analysis of small particulates suspended in slow moving flow medium such as the gas phase (the simulation is done in water), and to study the various aspects affecting particle size measurements.

\section{Principles of the PIV technique}

Microscopy is often used as a method of particle size analysis since it allows close observation and measurement of individual solid particles. However, these measurements cannot be carried out on the flowing or dynamic particulate. Most powder particles, gas bubbles or liquid droplets are moved by a primary fluid motion; consequently a method capable of analysing dynamic particulate is required. The introduction of quantitative laser sheet diagnostic techniques has made possible the non-intrusive measurement of the dynamic particulate characteristics. These include particles velocity, size and flux.

The PIV technique is a promising method, in terms of accuracy, resolution and reliability, for the instantaneous qualitative and quantitative measurements of a whole flow field. Its unique ability to extract quantitative information on steady or unsteady flow structure, made the technique useful and feasible in a wide range of industrial applications, such as turbo machinery, biology, medicine and environment [4]. 
The principle of the PIV method is to determine the flow velocity from the motion of tracer particles such as dust particles here. A plane sheet of laser light illuminates the flow area of interest and the light scattered from the tracer particles is recorded by means of a camera. The velocity and particle information is then extracted across the region of interest within a single, double or multiple exposures. Further processing can be carried out for flow visualisation and particle analysis [5].

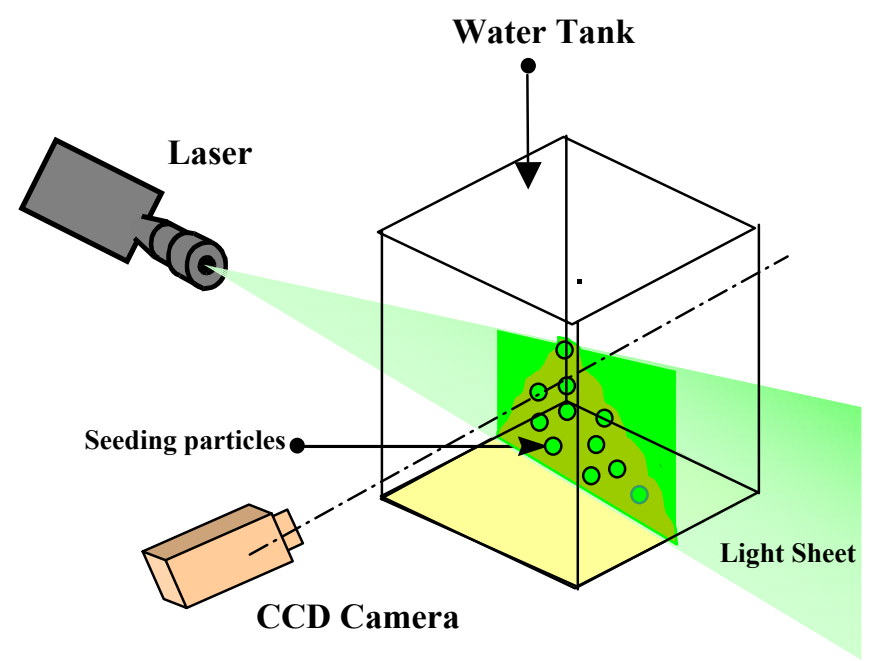

Figure 1: Experimental PIV test rig setup.

\section{Experimental rig setup}

The small scale test rig setup of the PIV system (figure 1), that have been build for simulation, consists of:

- A laser light source and its corresponding optics to create a light sheet within the flow of interests. Two types of lasers have been used: A Nd:Yag twin pulsed laser that gives $150 \mathrm{~mJ}$ pulses at $15 \mathrm{~Hz}$ frequency and a $1 \mathrm{~W}$ continuous wavelength laser.

- An optical recording device, which in this case is a charge-coupled device (CCD) camera used for capturing the image formed by the scattered light from the seeding. Two types of CCD cameras have been used: a Kodak ES 1.0 with a resolution of $1008 \times 1018$ array pixels and a $60 \mathrm{~mm}$ lens, and a PixeLink PL-A782 firewire CCD camera with a resolution of 6.6 Mega pixels. In both case Images have been acquired with 256 grey levels.

- A source of tracer particles to represent the particulate phase. Known size seeding particles $(10,30,100,250$ microns) with different densities in water have been used in order to simulate the various degree of particle pollution in PIV images. 
- A test rig consisting of a fish tank containing water. The water has been used to simulate the fluid medium with low velocity flow. A variable speed water pimp has been used in order to vary the velocity of the primary fluid medium.

\section{Results and discussion}

In order to develop the principals of a non-intrusive device, using the Particle Image Velocimetry (PIV) laser technique, for continuous measurements of dust particulate, a small scale test rig has been built to simulate low velocity fluid medium with known seeding particle.

The aim of these experimental tests is to process and analyse the particulate size and distribution ranging from few microns to many hundreds of microns. As it can be expected, the determination of the size of particle images is a crucial element in the accurate measurement and analysis of particle size.

In order to measure accurately these particles, a proper calibration procedure for such measurements is required. Therefore, several sets of images have been collected to study the various aspects affecting particle size measurements.

Three main parameters have been chosen for this study that affects greatly the size measurement of particles. These parameters are: fluid flow velocity, camera aperture and particulate size. Although, other parameters could have been chosen for study, such laser power and different types of lasers, however due to the high cost of these equipments, it was not possible at this stage to perform these tests. Several sets of images, with various parameter settings, have been acquired (Table 1). The total number of pictures acquired for this study is over 5000 images.

Table 1: List of the various parameters, ranges and acquired images.

\begin{tabular}{|c|c|c|}
\hline Parameters & Ranges & $\begin{array}{c}\text { Sets of acquired } \\
\text { images }\end{array}$ \\
\hline Fluid flow velocity $(\mathrm{cm} / \mathrm{s})$ & $0,10,20,30,40$, and 50 & 1600 \\
\hline Camera aperture & $3.5,5.6,8,11$, and 16 & 1200 \\
\hline Particulate size (microns) & $10,30,100$, and 250 & 1200 \\
\hline
\end{tabular}

From the figures below, it is quite clear to which extent the various settings affect the size measurement of particle images. By having the same aperture of the camera and the medium flow velocity, figure 2 illustrates the effects of the particle size has on the scattered light recorded by the camera. Although this result is expected, it can be noticed that the scattered light is not linear or with the same proportions compared to the respective particle sizes (10 $\mu \mathrm{m}$ (fig A) and $100 \mu \mathrm{m}$ (fig B)). This due to the fluid and particulate dynamics affecting the amount of scattered light from these particles. 


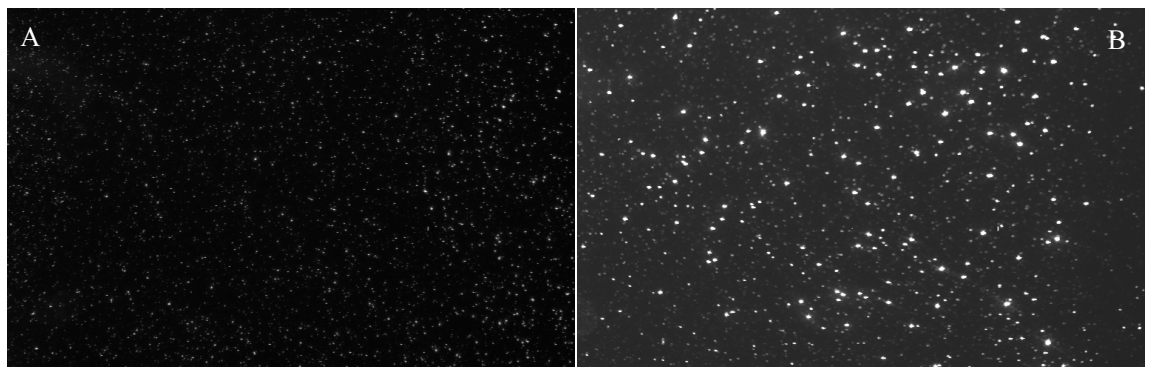

Figure 2: Images with the same velocity $(10 \mathrm{~cm} / \mathrm{s})$, same aperture (3.5), but with different particle sizes (10 $\mu \mathrm{m}$ (fig A) and $100 \mu \mathrm{m}$ (fig B)).

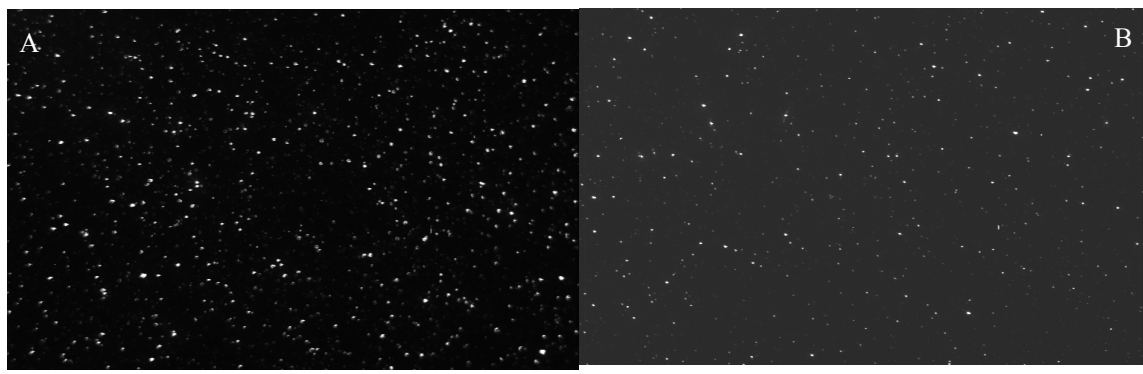

Figure 3: $\quad$ Images with the same particle size $(30 \mu \mathrm{m})$, same aperture (8), but with different velocity $(10 \mathrm{~cm} / \mathrm{s}$ (fig A) and $50 \mathrm{~cm} / \mathrm{s}$ (fig B))

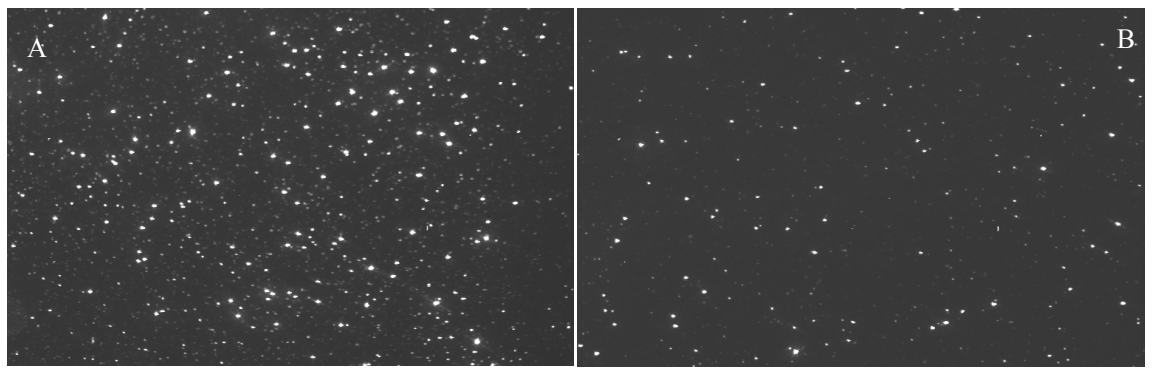

Figure 4: Images with the same velocity $(20 \mathrm{~cm} / \mathrm{s})$, same particle sizes $(100$ $\mu \mathrm{m})$, but with different apertures (3,5 (fig A) and 11 (fig B))

Figure 3 and Figure 4 illustrate as well the profound effect of the medium flow velocity and camera aperture on the amount of scattered light from particulates. This is due to the resident time of particles within the laser light 
sheet plane, and the amount of scattered light that the camera is collecting to produce particle images.

Further algorithms are required for qualitative analyse of the data in order to have a better understanding of the dynamics involved and to obtain more accurate size measurements of particles.

\section{Conclusion}

A small-scale test rig has been built to simulate an online emissions monitoring system that continuously measures microscopic particulate covering the size range of dust particles. The PIV technique has been used to scrutinise the flow events within the rig.

This paper describes the various settings put in place in order to achieve this goal. The proper equipments have been chosen to enable the assessment and analysis of small particulates suspended in slow moving flow medium such as the gas phase (the simulation is done in water), and to study the various aspects affecting particle size measurements. The medium flow velocity, camera aperture and particulate size parameters have been chosen for this study. The images collected showed the significant effects that all these parameters have on the size measurement of particulates.

All these images are currently being processed further for qualitative analyses. This will allow for a better understanding of the particulates dynamics and the parameters that can affect significantly the size measurement of particles and the calibration of such devices.

Environmental interest of such an application is very high. The reason being is that this analysis can be applied to any type of gas, albeit safe or dangerous gases.

\section{Acknowledgement}

An acknowledgement is due to Dr A. Aroussi, from The University of Nottingham (UK), for his valuable support to this project.

\section{References}

[1] James A, Jahnke C, Continuous Emission Monitoring, Environmental Engineering, John Wiley \& Sons, 2002.

[2] Raffel M., Willert C., Particle Image Velocimetry - A Practical Guide, Springer Publisher, 2001.

[3] Willert C.E, Gharib M, Digital particle Image Velocimetry, Experiments in Fluids, Volume 10, 1991.

[4] Raffel M, Willert C, Kompenhans J, Particle Image Velocimetry, Springer, New York, 1998.

[5] Menacer M, Aroussi A, Guendouz S, Adaptive contrast enhancement method for typical histogram configuration, IEE Electronics Letters, UK, Vol 35, No 15, July 1999, pp 1235. 